\title{
CLINICAL CASE OF A PATIENT UNDERGOING RADIUM-223 TREATMENT FOLLOWING TREATMENT WITH ABIRATERONE ACETATE AND ENZALUTAMIDE
}

\author{
M.E. Jiménez Romero*, S. Díez Farto, J.C. Navarro Serrato, E. Canelón Castillo, I. Revelo Cadena \\ Urological Department of the University Hospital of Puerto Real, Cádiz 11510, Spain
}

\begin{abstract}
Objective: Over the last decade, significant advances have been made in the development of therapies for patients with metastatic castration-resistant prostate cancer. Abiraterone and enzalutamide were approved as treatments based on data supporting improved overall survival compared to placebo. Radium-223 became the first approved radiopharmaceutical which decreased skeletal-related events, palliated pain, and showed improved overall survival in symptomatic patients with castration-resistant prostate cancer and bone metastasis only. Materials and Methods: We present the case of an eighty-two year old man with metastatic castrationresistant prostate cancer who was treated with sequential therapy (abiraterone - enzalutamide - radium 223). The sequencing and treatment used for our patient was viable because of his clinical characteristics, which have allowed for longer survival time with an acceptable quality of life. These actions must be agreed on by the Multidisciplinary Tumour Board, in order to optimize the use of available courses of treatment. Results: The treatment of these patients is changing rapidly, but many questions remain regarding the optimal sequencing of the available drugs. Sequential or concomitant use of the next generation hormonal agents - abiraterone and enzalutamide - cannot currently be recommended. Data regarding the safety of concomitant abiraterone, enzalutamide or denosumab with radium-223 is reassuring and timely. However, we cannot advocate the general use of combined radium-223 therapy at this time, irrespective of prior therapy. Conclusion: A better understanding of active mechanisms, the genetic characteristics of each metastatic castration-resistant prostate cancer and the development of new prognostic and predictive biomarkers will help determine sequencing or different combination treatments for each individual patient.
\end{abstract}

Key Words: castration-resistant prostate cancer, treatment sequence, abiraterone acetate, enzalutamide, radiopharmaceutic.

Prostate cancer $(\mathrm{PC})$ is the second most commonly diagnosed cancer, and is the fifth leading cause of cancer death in men [1]. Most of the men who are diagnosed with PC present with apparent localized disease. Despite initial treatment, some of these men will go on to have disease progression, firstly presenting with biochemical recurrence even if there is no clinical or radiographic evidence of disease. Then, many continue to progress and develop distant metastatic disease. Other patients present with metastatic disease de novo at the time of the first diagnosis. Treatment for advanced disease usually begins with androgen deprivation therapy. The majority of cases initially respond to androgen-deprivation, progressing towards castration-resistance. Most patients will develop metastases - bone metastasis being the most frequently observed - and an independent poor prognostic factor [2]. Until recently, the only treatment for these patients that had proven successful in increasing overall survival rates was docetaxel. In the last decade, significant progress has been made in the development of new agents against metastatic castration-resistant prostate cancer (MCRPC) [3]. Treatments based on abiraterone acetate (AA) [4] and enzalutamide (ENZ) [5] were approved after the publication of data which demonstrated an increase of the overall survival rates, when compared to a pla-

Submitted: January 11, 2018.

*Correspondence: E-mail: miguelefrenjimenez@gmail.com Abbreviations used: $\mathrm{AA}$ - abiraterone acetate; $\mathrm{CT}$ - computerized tomography; ENZ - enzalutamide; mCRPC - metastatic castration-resistant prostate cancer; PC - prostate cancer; PSA prostate-specific antigen; SRE - skeletal-related event. cebo group, simultaneously reducing the time period to the first skeletal-related event (SRE) and - in the case of AA treatment - reducing pain. In the last few years, the high efficiency and low toxicity of both treatments, coupled with the experience acquired, have changed the conditions of our patients suffering from mCRPC. Furthermore, the therapeutic algorithm of these patients has changed recently with the introduction of radium-223, the first approved radiopharmaceutical which has shown an improvement in pain control, reduced SREs and increased survival rates in symptomatic or minimally symptomatic patients with $\mathrm{mCRPC}$ and bone metastasis.

\section{MATERIALS AND METHODS}

Eighty-two year old male with existing history of high blood pressure, being treated with enalapril; and of hypercholesterolemia, being treated with simvastatin. Ex-smoker of 10 cigarettes a day for many years. In August 2009, at 75 years of age, the patient was diagnosed with a sigmoid colon adenocarcinoma (stage IIA) requiring surgery (laparoscopic sigmoidectomy) and adjuvant chemotherapy (since then, Oncology has carried out several follow-up consultations with no recurrence).

In May 2010, the patient attended our practice following a referral from Oncology for a symptomatology assessment of the lower urinary tract, presenting with high prostate-specific antigen (PSA) levels $(24.20 \mathrm{ng} / \mathrm{ml})$. The rectal examination revealed a prostate of increased size and mass. A transrectal prostate biopsy was carried out, the results of which confirmed a prostate adenocarcinoma with a Gleason score of $8(4+4$, bilateral) with marked perineural invasion 
and extension to the prostate tissue on both lobes. The extension study, carried out by means of computerized tomography $(\mathrm{CT})$, revealed an extracapsular extension, but no visceral or lymph node metastases. The bone scan provided findings consistent with metastatic bone disease (in the right sacroiliac region; clinical stage cT3b N0 M1b).

Given these results andaPC diagnosisatanadvanced metastatic stage, continuous androgen-deprivation therapy was initiated (six-monthly luteinizing-hormone releasing-hormone agonist) + zoledronic acid (4 mg administered intravenously on a monthly basis, for 18 months) + calcium and vitamin D supplements. Initially, monitoring took place at a follow-up visit three months later and every six months after that.

A course of monotherapy with a six-monthly agonist was initiated in July 2010, prompting a rapid biochemical response and a reduction of PSA levels, which reached their lowest point in December 2011 at $0.40 \mathrm{ng} / \mathrm{ml}$. Testosterone remained at castration level. In March 2013, PSA levels increased up to $2.78 \mathrm{ng} / \mathrm{ml}$, which prompted further hormonal treatment yielding a biochemical response lasting up to August 2013 (PSA levels of $5.78 \mathrm{ng} / \mathrm{ml}$ ). Consequently, a new radiological assessment with a CT scan was requested, which ruled out visceral metastases, while the bone scan revealed new affected areas in the left sacroiliac region and on the right ischium (Fig. 1). Following the diagnosis of mCRPC, the patient was examined in a specific consultation which included a full evaluation. The response to the 35-month androgendeprivation therapy presented a score of 0 according to the ECOG scale; the patient was asymptomatic and did not require analgesia ( 0 , according to the brief pain inventory - short form), although the patient did complain of mild asthenia.

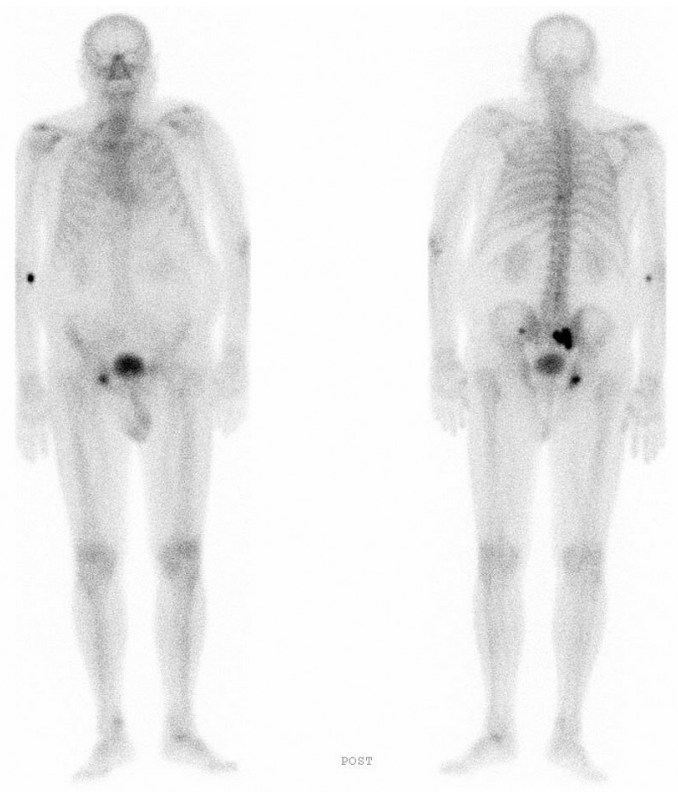

Fig. 1. Bone scan revealing affected areas in the left sacroiliac region and right ischium

In light of all these considerations, treatment with AA (four 250 mg capsules a day +10 mg predni- sone daily) was initiated in September 2013. At the same time, it was decided that treatment for bone metastasis be resumed by prescribing denosumab (120 mg as a monthly subcutaneous injection). In June 2014, after 9 months of treatment, PSA levels reached their lowest point, at $0.25 \mathrm{ng} / \mathrm{ml}$, with alkaline phosphatase levels remaining within normal limits.

The patient remained stable, without clinical or analytical changes, no side effects, and with quarterly check-ups (including a new CT scan and a bone scan 15 months after the beginning of treatment) until August 2015. At that time, PSA levels, which had remained stable thus far, increased to $8.30 \mathrm{ng} / \mathrm{ml}$ and alkaline phosphatase levels increased to $154.5 \mathrm{U} / \mathrm{l}$. A mild clinical deterioration of the patient's health was observed and first-line analgesic treatment (paracetamol) was prescribed with good results.

At the beginning of December 2015, after 27 months of treatment, PSA levels were $30.43 \mathrm{ng} / \mathrm{ml}$, with significant asthenia and pain controlled by means of the prescribed analgesic. Alkaline phosphatase levels reached $342 \mathrm{U} / \mathrm{I}$ and a new extension study with a CT scan and bone scan demonstrated advanced bone metastasis (Fig. 2, 3).

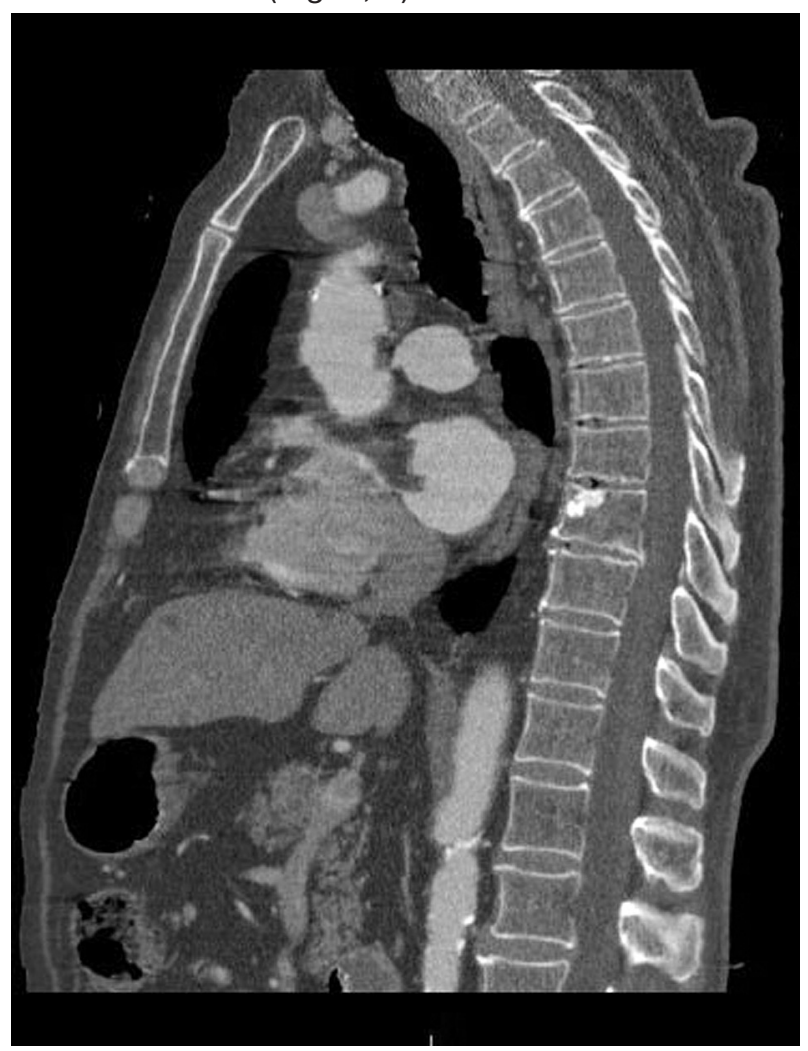

Fig. 2. CT scan showing advancement of the bone metastasis

In February 2016, AA was suspended and it was decided that treatment consisting of ENZ (four $40 \mathrm{mg}$ capsules daily) be initiated. The patient presented with more acute asthenia and worsening hip pain, which necessitated second-line analgesic treatment (tramadol). Two months into the ENZ treatment, and due to the limited clinical benefits observed associated with a reduced quality of life due mainly to pain - this treatment was suspended and new radiological tests were requested, with a view to starting 


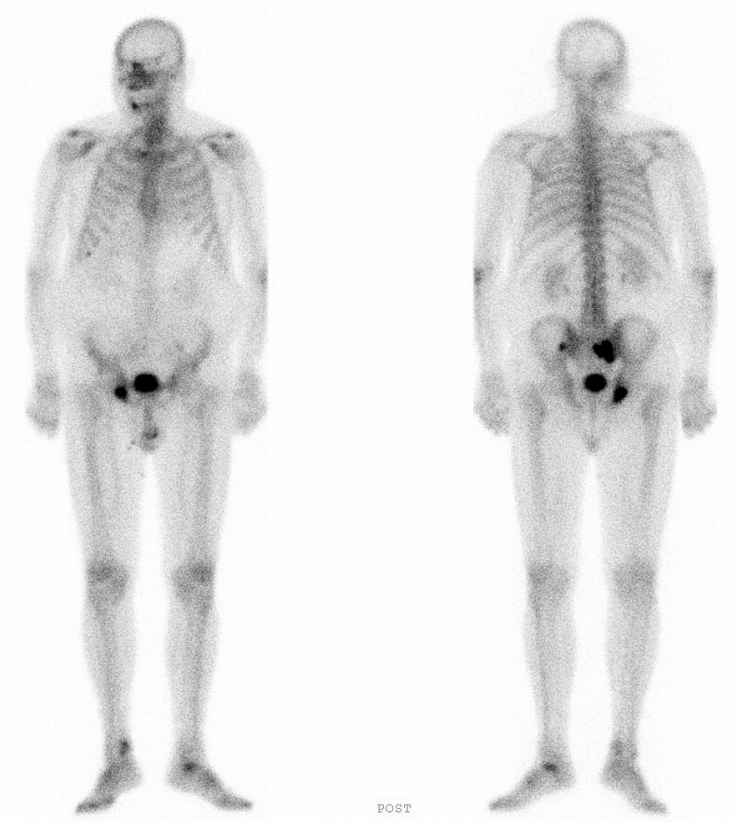

Fig. 3. New bone scan showing advancement of the bone metastasis

treatment based on radium-223. The CT scan of the chest, abdomen and pelvis displayed advancement of the bone metastasis without visceral or lymph node disease (Fig. 4). A basal haematological evaluation was carried out including an absolute neutrophil and platelet count, as well as an assessment of hepatic and renal function, which did not contraindicate the start of treatment. In this context of symptomatic bone disease without visceral or lymph node disorders, it was decided that treatment based on radium-223 be initiated.

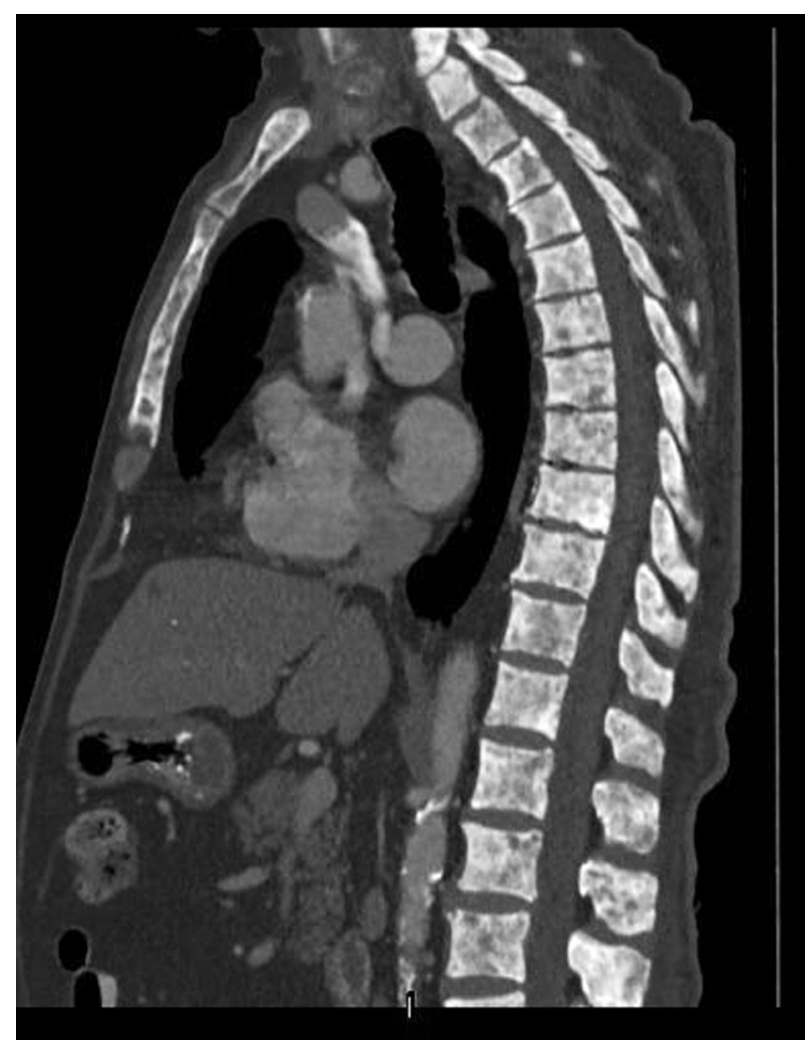

Fig. 4. Chest, abdomen and pelvis CT scan; advanced bone metastasis; lack of visceral or lymph node metastases
Close cooperation with the Nuclear Medicine Service was established, and a joint follow-up schedule was agreed upon, consisting of a full blood test before each dose of radium-223. The patient completed six full one-month cycles of treatment. He received the first dose in May 2016, having previously suspended treatment with calcium and vitamin D supplements. Coinciding with the administration of the first dose, the patient reported a slight increase of polyostotic bone pain, corresponding to a flare effect described in the pivotal study (ALSYMPCA) [6]. Four weeks later, following the administration of the second dose, the blood tests detected an increase of PSA levels and a reduction of alkaline phosphatase levels, showing pain relief and good tolerance towards the treatment. Therefore, the therapeutic programme was continued. Before the third dose, the patient suffered from anaemia $(\mathrm{Hb}=8.2 \mathrm{~g} / \mathrm{dl})$ which prompted the decision to carry out two transfusions of red blood cell concentrates. During the rest of the dosage, the patient reported two isolated cases of diarrhoea. Altogether, the patient showed very considerable improvement in terms of pain relief and did not require further analgesic treatment. Notwithstanding, PSA levels increased up to $421 \mathrm{ng} / \mathrm{ml}$, at the date of the final dose, whereas alkaline phosphatase levels fell considerably ( $187.7 \mathrm{U} / \mathrm{I})$ without returning to normal levels.

In November 2016, a reassessment was carried out after the course of treatment had been completed which presented a mild clinical deterioration. The patient continued to take a six-monthly agonist with a calcium and vitamin D supplement, which was re-established following the final dose of the radiopharmaceutical. PSA levels remained stable $(435 \mathrm{ng} / \mathrm{ml})$ and alkaline phosphatase levels increased up to $545 \mathrm{U} / \mathrm{l}$.

At the end of December 2016, the bone and CT scans displayed further progression of the bone metastasis (Fig. 5). The patient was re-admitted twice with anaemia, requiring a blood transfusion. At the end of February 2017, they were admitted again for anaemia, requiring a transfusion $(\mathrm{Hb}=6.7 \mathrm{~g} / \mathrm{dl})$, and predominantly evening asthenia with bone pain controlled by means of a second-grade analgesic treatment (tramadol). The monthly follow-up appointments were continued (for the control of the anaemia and the pain).

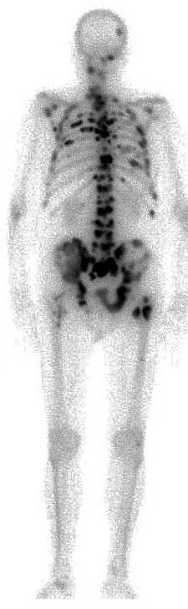

Fig. 5. Diffuse bone metastasis on bone scintigraphy 
Eighty-three months after the PC diagnosis and as of March 2017, the patient has an acceptable quality of life. Following completion of the treatment, we have observed marrow toxicity and requested transfusions every four to five weeks in order to treat grade 3 anaemia. The patient reports general bone pain which currently require third-line analgesic treatment (morphic drugs). Considering all the above, it was decided that systemic treatment with chemotherapy be ruled out.

\section{DISCUSSION}

The emergence of these new highly effective therapies poses a significant clinical challenge; in this regard, the current debate is trying to position radium-223 within the therapeutic algorithm of $\mathrm{mCRPC}$ [7]. The question is whether to administer it before, during the course of the disease, concomitant with new hormonal therapies or, on the contrary, to wait until the patient progresses to a treatment with chemotherapy, ENZ or AA. Furthermore, if the option chosen is to combine it with AA or ENZ, it would be essential to know the efficacy and safety of this combination.

Sequencing is being trialled with the hope of obtaining a cumulative benefit for survival. In the pre-chemotherapy setting, the information currently available is based on retrospective studies of very small series and a highly heterogeneous patient population [8]. However, the efficacy of this practice has not been backed up by data derived from clinical trials, and evidence from potential cross-resistance reinforces the debate surrounding the best sequence to use in order to maximize the benefits. This situation becomes more difficult given evidence showing the possibility of administering hormonal agents in patients who have not undergone chemotherapy and administering new chemotherapeutic agents in patients sensitive to hormone treatment. Thus, a thorough study is required, including the characteristics of each patient and their disease, as well as safety profiles and the efficiency of each drug, in order to be able to find the best treatment sequencing for each individual, whether as monotherapy or as in combination $[9,10]$. For example, if a patient with an asymptomatic case of mCRPC undergoing concomitant treatment with docetaxel and radium-223 showed progression to the bone, the possible options available later in terms of bone pain treatment are drastically reduced. Definitive data will be published soon regarding the safety and efficiency of re-treatment with radium-223 in patients who have previously been administered with docetaxel. These results may serve to clarify some of our doubts.

In terms of a combination of treatments, Saad et al. [11] have recently published a phase $3 b$ trial analyzing the safety and efficiency of radium-223 and other concomitant therapies in patients with CPRC. It includes a total of 606 Caucasian symptomatic or asymptomatic males, over 18 years old, with a prevalence of bone disease and at least two bone metastases. Patients with visceral disease were excluded but not those with lymph node metastases. Patients were to receive six intravenous injections of radium-223 every four weeks in $50 \mathrm{kBq} / \mathrm{kg}$ doses. Other concomitant therapies were permitted, such as AA, ENZ or denosumab. The primary endpoints of the study were overall survival and safety. Only $58 \%$ of patients were administered with the six injections of radium-223. After a median follow-up period of 7.5 months, the authors reported an improvement of overall survival rates in patients taking radium-223 in combination with either $A B I$, $E N Z$, ENZ and $A B I$, or denosumab. The same benefit was not observed in patients who were administered with radium-223 and bisphosphonates. Tolerance for the treatment was good with only $21 \%$ of patients suspending the treatment due to side effects. The most common side effect was grade 3 anaemia (12\%) and grade 3-4 combined cytopenia (anaemia, thrombocytopaenia, leukopenia and neutropenia), which were observed in $20 \%$ of subjects. Given the relatively short follow-up period ( 7.5 months), the possibility of delayed or persistent marrow toxicity under these combinations is still to be determined.

We believe that the number of radium-223 doses and infusions needed in this combined therapy could be lower than those currently approved for monotherapy. At the same time, it is yet to be determined whether this combination could lead to the occurrence of a form of PC which is highly resistant to the treatment, given the potential biological alteration of the tumor or the development of resistance (AR-V7) [12].

Our patient comfortably met the inclusion criteria set up for the pivotal trial (ALSYMPCA) [1]. Following a good response to AA treatment, the sequential ENZ therapy did not prove to be effective, leading to rapid clinical, biochemical and radiological progression. After this, the patient began radium-223 treatment with a good clinical response to pain, which was reduced after the first dose. Furthermore, no bone-related events arose, which was of considerable benefit to the patient's quality of life. Moreover, the side effect profile during the treatment was acceptable and easily managed by the clinician.

\section{CONCLUSIONS}

Although treatment for mCRPC is changing rapidly, there are still many open questions regarding the optimal treatment sequence. The sequential or combined use of AA and ENZ is currently not recommended. We chose this particular sequencing for the patient because of his characteristics. At said time, there was little evidence available regarding the AA - ENZ Sequencing. Normally, unltil solid evidence is available, this type of sequencing not apply on our patients.

Radium-223 is a therapeutic option for mCRPC patients, both as an initial treatment or in cases of resistance towards other treatments, as its action mechanism suggests few chances of crossed resistance to other agents. The results obtained demonstrate that radium-223 can be safely combined with AA or ENZ, 
which are standard treatments for MCRPC patients as has been proven by this particular case - with little and manageable side effects.

At the same time, these results can be applied to asymptomatic patients, who were not analyzed as part of the ALSYMPCA pivotal study. In our opinion, radium-223 should feature in the early stages of our therapeutic algorithm, and be administered before patients develop extra-osseous disease.

The emergence of new therapies against mCRPC is expected soon, which means that the challenge will be to learn how to combine and sequence these agents. Ongoing studies, along with the additional information expected from the studied biomarkers, will help us refine our selection of suitable treatments [13].

\section{REFERENCES}

1. American Cancer Society. Global cancer facts and figures. $3^{\text {rd }}$ ed. Atlanta: American Cancer Society, 2015.

2. Emrich LJ, Priore RL, Murphy GP, et al. Prognostic factors in patients with advanced stage prostate cancer. Cancer Res 1985; 45: 5173-9.

3. Zafeiriou Z, Jayaram A, Sharp A, et al. Managing metastatic castration-resistant prostate cancer in the prechemotherapy setting: a changing approach in the era of new targeted agents. Drugs 2016; 76: 421-30.

4. de Bono JS, Logothetis CJ, Molina A, et al. Abiraterone and increased survival in metastatic prostate cancer. N Engl J Med 2011; 364: 1995-2005.
5. Scher HI, Fizazi K, Saad F, et al. Increased survival with enzalutamide in prostate cancer after chemotherapy. N Engl J Med 2012; 367: 1187-97.

6. Parker C, Nilsson S, Heinrich D, et al. Alpha emitter radium-223 and survival in metastatic prostate cancer. $\mathrm{N}$ Engl J Med 2013; 369: 213-23.

7. Yeku O, Slovin SF. Radium-223 and concomitant therapies: prospects and prudence. Transl Androl Urol 2016; 5: 968-70.

8. Azad AA, Eigl BJ, Murray RN, et al. Efficacy of enzalutamide following abiraterone acetate in chemotherapy-naive metastatic castration-resistant prostate cancer patients. Eur Urol 2015; 67: 23-9.

9. Caffo O, Lunardi A, Trentin C, et al. Optimal sequencing of new drugs in metastatic castration-resistant prostate cancer: dream or reality? Curr Drug Targets 2016; 17: 1301-8.

10. Maines F, Caffo O, Veccia A, et al. Sequencing new agents after docetaxel in patients with metastatic castrationresistant prostate cancer. Crit Rev Oncol Hematol 2015; 96: 498-506.

11. Saad F, Carles J, Gillessen S, et al. Radium-223 and concomitant therapies in patients with metastatic castrationresistant prostate cancer: an international, early access, open-label, single-arm phase 3b trial. Lancet Oncol 2016; 17: $1306-16$.

12. Antonarakis ES, Lu C, Wang $\mathrm{H}$, et al. AR-V7 and resistance to enzalutamide and abiraterone in prostate cancer. N Engl J Med 2014; 371: 1028-38.

13. Handy CE, Antonarakis ES. Sequencing treatment for castration-resistant prostate cancer. Curr Treat Options Oncol 2016; 17: 64. 